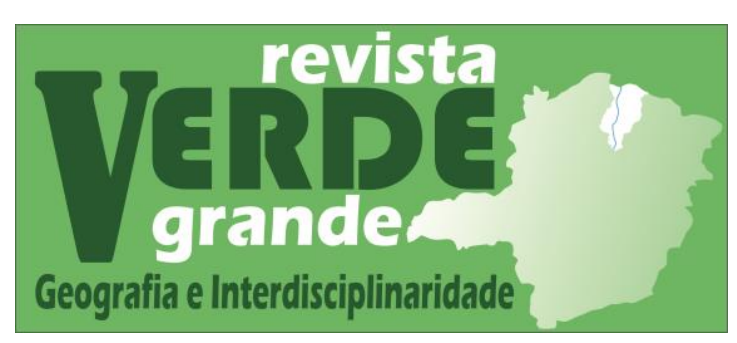

Volume 2, no. 1 (2020)

ISSN: 2675-2395

https://doi.org/10.46551/rvg267523952020198119

\title{
ÍNDICE RELATIVO DE QUALIDADE DE VIDA DA REGIÃO GEOGRÁFICA INTERMEDIÁRIA DO NORTE DE MINAS GERAIS/MG
}

\author{
Relative Index Of Quality Of Life The Intermediate Geographic Of The North Of Minas Gerais/MG
}

Junia de Souza Silva ${ }^{1}$ https://orcid.org/0000-0002-9372-0626

\footnotetext{
${ }^{1}$ Mestranda em Desenvolvimento Econômico e Estratégia Empresarial da Universidade Estadual de Montes Claros - UNIMONTES. E-mail: junia.ssilva@yahoo.com.br
}

\begin{abstract}
Resumo
Analisar o desenvolvimento de uma região colabora para realizações e aperfeiçoamento de políticas públicas que sejam engajadas nas especificidades de individual de cada área. Através de dados secundários disponibilizados pelo IBGE no Censo de 2010, esta pesquisa se atentou a construir o Índice Relativo de Qualidade de Vida (IRQV) dos municípios da região geográfica intermediária do Norte de Minas Gerais/MG, anteriormente denominada de Mesorregião do Norte de Minas Gerais/MG. Através das variáveis locacionais e optando pela realização da análise fatorial para estudar a correlação entre estas, foi possível verificar que o município de Montes Claros apresenta um índice maior de desenvolvimento que os demais municípios do conglomerado. Em contraponto, o município de São João das Missões apresentou o pior índice entre os oitenta e nove municípios componentes deste estudo. Revela-se ainda a necessidade de outros estudos regionais sejam realizados para verificação da continuidade em crescimento regional, uma vez que há uma lacuna temporal de dez anos desde a última realização do Censo brasileiro.
\end{abstract}

Palavras-chave: Desenvolvimento Regional. IRQV. Norte de Minas Gerais.

\begin{abstract}
Analyzing the development of a region contributes to the realization and improvement of public policies that are engaged in the specificities of each individual in each area. Through secondary data made available by IBGE in the 2010 Census, this research attempted to build the Relative Quality of Life Index (IRQV) of the municipalities in the intermediate geographic region of Northern Minas Gerais/MG, formerly called the Mesoregion of Northern Minas Gerais/MG. Through the locational variables and opting for the factorial analysis to study the correlation between them, it was possible to verify that the municipality of Montes Claros has a higher development index than the other municipalities in the conglomerate. In contrast, the municipality of São João das Missões had the worst index among the eighty-nine municipalities included in this study. It also reveals the need for further regional studies to be carried out to verify the continuity in regional growth, since there is a ten-year time gap since the last Brazilian Census.
\end{abstract}

Keywords: Regional Development. IRQV. North of Minas Gerais. 


\section{Introdução}

A estrutura econômica brasileira modificou-se no decorrer dos anos, principalmente a partir dos anos de 1990 marcando "por um processo de abertura comercial abrangente, que se iniciou no governo Collor e se estendeu até o governo Fernando Henrique” (AVERBURG, 1999, p. 45). Na década seguinte, a "adoção de programas sociais focalizados na redução das desigualdades socieconômicas e na melhoria das condições de vida da população foi intensificada" (CARDOSO et al., 2015, p. 348).

Avanços socioeconômicos foram realizados, porém "as desigualdades regionais persistem, representando um ponto crucial que impede o processo de desenvolvimento" (CARDOSO et al., 2015, p. 348). Porém, no processo de integração do desenvolvimento elenca diversos aspectos, dentre eles a definição sistêmica da economia sobre a distinção entre desenvolvimento econômico e crescimento econômico.

O desenvolvimento econômico, segundo Bresser-Pereira (2008, p. 1) é definido como a "sistemática acumulação de capital e de incorporação do progresso técnico ao trabalho e ao capital que leva ao aumento sustentado da produtividade ou da renda por habitante e, em consequência, dos salários e dos padrões de bem-estar de uma determinada sociedade".

Diante disso, os "trabalhos têm atribuído especial atenção à construção de indicadores de desenvolvimento que permitam a caracterização do nível de bem-estar a nível estadual e/ou municipal" (CARDOSO et al., 2015, p. 350) são tão importantes para mensurar os avanços que estão sendo elencados ao passar dos anos.

O propósito deste é o de elaborar o índice relativo de qualidade de vida nos Municípios da região geográfica intermediária do Norte De Minas/MG, tendo como base de dados o Censo disponibilizado pelo Instituto Brasileiro de Geografia e Estatística - IBGE do ano de 2010, podendo, após a criação do índice, verificar quais municípios possuem indicadores com menor e com maior grau de desenvolvimento econômico para a região geográfica do norte mineira no período analisado.

Para isso, a metodologia a ser aplicada foi a técnica de estatística multivariada, análise fatorial, para construção do indicador relativo à qualidade de vida denominado Índice Relativo de Qualidade de Vida (IRQV) dos municípios do conglomerado, além de se correlacionar à autores que já apresentaram trabalhos relativos a temática abordada deste artigo. 


\section{Desenvolvimento Econômico e Crescimento Econômico}

Alguns economistas realizam diferenciação entre o desenvolvimento econômico e crescimento econômico. Schumpeter (1982, p. 11) atribui diferenças entre crescimento e desenvolvimento descrevendo que "nem o mero crescimento da economia, representado pelo aumento da população e da riqueza, será designado aqui como um processo do desenvolvimento".

Ao desenvolver uma economia autossustentável, significa que os Estados deverão formular estratégias permitindo-lhes o sucesso de integração no mercado global, sendo este capaz de acumular capital e progresso tecnológico nacional (BRESSER-PEREIRA, 2008, p. $01)$.

Na concepção de Veiga, (2005, p. 80) o desenvolvimento é apresentado como:

[...] o desenvolvimento pode permitir que cada indivíduo revele suas capacidades, seus talentos e sua imaginação na busca da auto-realização e da felicidade, mediante esforços coletivos e individuais, combinação de trabalho autônomo e heterônomo e de tempo gasto em atividades não econômicas.

Já o crescimento econômico além de ser baseado nas concepções de aumento da capacidade produtiva, sendo "concentrador de renda ou adverso" (Bresser-Pereira, 2008, p. 03), estruturam questões econômicas como o produto interno bruto (PIB) e o produto nacional bruto (PNB).

Apesar de serem realizadas distinções entre os dois conceitos, para Bresser-Pereira (2008, p. 04), ambas as expressões podem ser utilizadas basicamente como sinônimas, apresentando um apresso maior pelo desenvolvimento, uma vez que pode ser aplicado como "algo mais amplo e melhor".

Para a economia nacional, considerando expressamente que desenvolvimento econômico e crescimento econômico não são expressões sinônimas, a estrutura e o funcionamento econômico dependem de decisões e de políticas voltadas para a realidade daquela economia (FURTADO, 2008, p. 33).

Obstante disso, o "conhecimento integrado das estruturas sociais e história de como estas estruturas sociais foram posicionadas são de importância preciosa tanto quanto no estudo das relações de poder geradas nas relações de poder, quanto no estudo daquela economia" (FURTADO, 2008, p. 33).

Ao levantar a temática de que o crescimento está associado "à melhoria das condições de vida da população, o PIB per capita passou a ser, a partir dos anos 1950, o indicador clássico de desenvolvimento em diversos países do mundo" (CARDOSO et al., 2015, p. 349). 
Porém, somente elencar prerrogativas de desenvolvimento interligadas o PIB per capita, mostra uma limitação, mesmo que analises consistentes sobre esta variável seja de fácil entendimento e de comparabilidade.

\title{
Caracteristicas Regionais: Mesorregiões e Microrregiões
}

Diniz e Batella (2005, p. 60), difundem a terminologia de região como estando diretamente "associado à ideia de diferenciação de áreas, ou seja, à aceitação de que a superfície da terra é formada por áreas diferentes entre si”. Este escopo proporciona a concepção de que mesmo distintas cada região conterá parte de um todo havendo "tantas regiões quantos forem os critérios adotados e o objetivo da regionalização".

O dinamismo do desenvolvimento capitalista no Brasil trouxe inevitavelmente a “desigualdade da organização espacial que comporta diferentes formas de subordinação do trabalho ao capital e pela atuação crescente do papel do Estado naquele processo" (INSTITUTO BRASILEIRO DE GEOGRAFIA E ESTATÍSTICA, 1990, p. 07).

De 1089 até o ano de 2017, o Instituto Brasileiro de Geografia e Estatística - IBGE subdividia o Estado Brasileiro de Minas Gerais/MG em 12 mesorregiões e 66 microrregiões ${ }^{2}$. As mesorregiões eram subdivisões realizadas pelo IBGE nos estados brasileiros para áreas geográficas que apresentavam características econômicas e sociais similares, além de outros aspectos apresentados pelo instituto:

\begin{abstract}
Entende-se por mesorregião uma área individualizada em uma Unidade da Federação que apresenta formas de organização do espaço geográfico definidas pelas seguintes dimensões: o processo social como determinante o quadro natural como condicionante e a rede de comunicação e de lugares como elemento da articulação espacial. Estes três dimensões possibilitam que o espaço delimitado como mesorregião tenha uma identidade regional. Esta identidade é uma realidade construída ao longo do tempo pela sociedade que aí se formou. $\mathrm{O}$ conhecimento da realidade espacial brasileira evidencia que o Agreste a Mata e o Sertão Nordestinos; o Sul de Minas Gerais; o Triangulo Mineiro; a Campanha Gaucha; as áreas coloniais Antiga e Nova do Rio Grande do Sul; o Vale do Itajaí; o Norte do Paraná; o Pantanal Mato-grossense; a Bragantina são unidades espaciais identificadas como mesorregiões (IBGE, 1990, p. 08, grifos do autor).
\end{abstract}

As microrregiões, conforme dispositivo constitucional vigente no artigo $25, \S 3^{\circ}$, consiste em um agrupamento de municípios limítrofes, cuja divisão tem por finalidade organizar com o objetivo de facilitar o planejamento e as execuções das políticas de gestão local. Além disso, a organização segue critérios também exemplificados pelo IBGE:

Informação
<https://www.mg.gov.br/sites/default/files/paginas/arquivos/2016/ligminas_10_2_04_listamesomicro.pdf $>$.

em Acesso em 02/01/2020. 
A organização do espaço microrregional foi identificada também pela vida de relações ao nível local, isto é pela interação entre as áreas de produção e locais de beneficiamento e pela possibilidade de atender às populações através do comércio de varejo ou atacado ou dos setores sociais básicos. Assim estrutura da produção para identificação das microrregiões é considerada em sentido totalizante constituindo-se pela produção propriamente dita distribuição, troca e consumo incluindo atividades urbanas e rurais. Dessa forma ela expressa a organização do espaço a nível micro ou local (IBGE, 1990, p. 08, grifos do autor).

A partir dessas estruturas, o modo de divisão territorial em mesorregiões e microrregiões estabeleceram estruturas que perduraram até o ano de 2017, quando o instituto reestruturou as subdivisões territoriais apresentando conceitos que, seguindo o próprio órgão, foca nas articulações apresentadas por cada região ${ }^{3}$.

A nova divisão territorial incorpora as mudanças no cenário brasileiro em duas escalas, sendo a primeira divisão nomeada região geográfica intermediária e a segunda região geográfica imediatas, cujas nomenclaturas anteriormente eram conhecidas como mesorregiões e microrregiões respectivamente.

Esta nova forma de divisão do território não altera ou substitui a divisão políticoadministrativa dos municípios, das Unidades Federativas e das Grandes Regiões. Portanto, serve apenas de um adicional na "construção do conhecimento geográfico" devido às mudanças ocorridas nas últimas décadas nos espaços regionais brasileiros (AGÊNCIA DE NOTÍCIAS IBGE, 2020).

\section{Região Geográfica Intermediária do Norte de Minas/MG.}

As divisões oficiais do Estado de Minas Gerais/MG são realizadas pelo Instituto Brasileiro de Geografia e Estatística - IBGE e pela Fundação João Pinheiro - FJP. O IBGE foi criado com o intuito de institucionalizar à divisão regional do território brasileiro (DINIZ, 2005, p. 63). Já a FJP desenvolve estudos sobre o território mineiro para melhor compreendêlo devido ao seu tamanho e sua diversidade espacial.

A região geográfica intermediária norte mineira compõe uma das doze antigas mesorregiões geograficamente divididas pelo IBGE. As mesorregiões do estado de Minas Gerais/MG são: Noroeste de Minas, Norte de Minas, Jequitinhonha, Vale do Mucuri, Triângulo Mineiro/Alto do Paranaíba, Central Mineira, Metrolopolitana de Belo Horizonte,

\footnotetext{
${ }^{3}$ Informação Disponível em <https://agenciadenoticias.ibge.gov.br/agencia-sala-de-imprensa/2013-agencia-denoticias/releases/10515-ibge-divulga-nova-divisao-territorial-com-foco-nas-articulacoes-regionais $>$. Acesso em 02/01/2020.
} 
Vale do Rio Doce, Oeste de Minas, Sul/Sudoeste de Minas, Campo das Vertentes e Zona da Mata, conforme determinadas no mapa 1:

Mapa 1: Mesorregiões de Minas Gerais/MG.

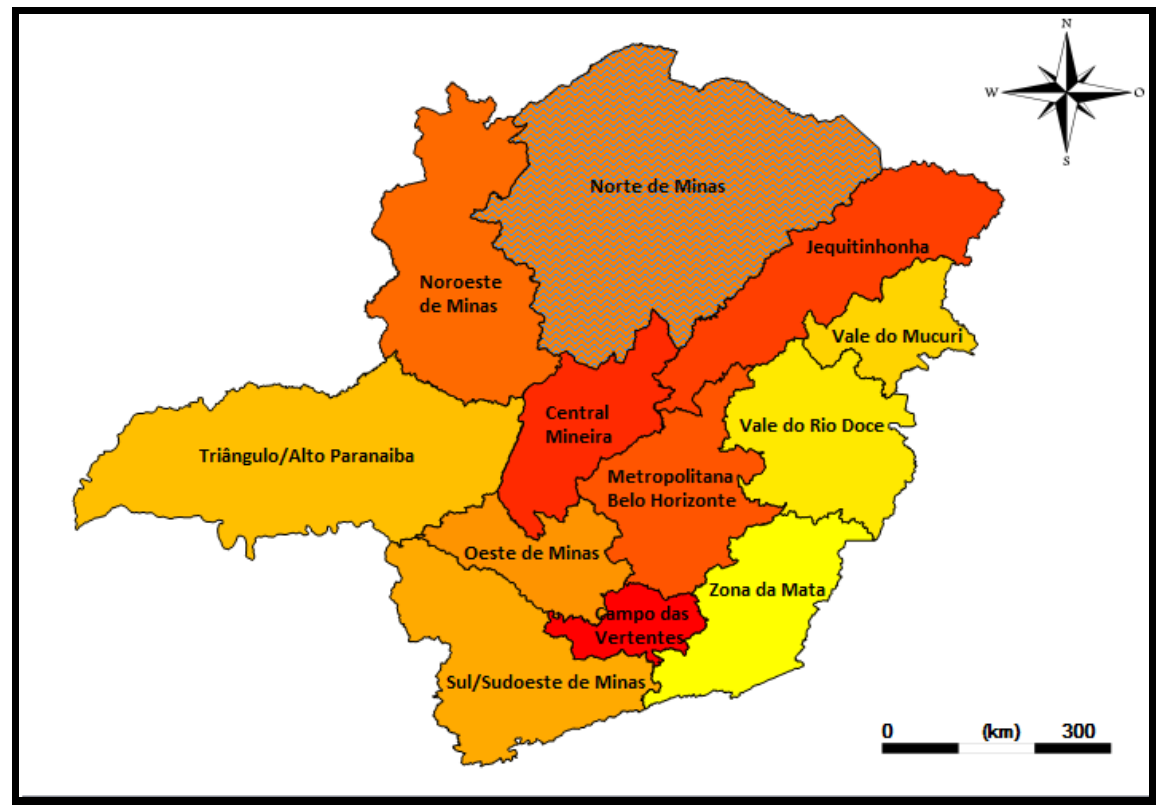

Fonte: Elaborado pela autora. Software IpeaGEO.

A região geográfica intermediária do Norte Mineiro é composta por 89 municípios sendo eles compostos no Quadro 01:

Quadro 1: Municípios Integrantes da Região Intermediária do Norte de Minas.

\begin{tabular}{llll}
\hline & & Municípios \\
\hline Águas Vermelhas & Glaucilândia & Matias Cardoso & Rubelita \\
Berizal & Grão Mogol & Mato Verde & Salinas \\
Bocaiúva & Guaraciama & Mirabela & Santa Cruz de Salinas \\
Bonito de Minas & Ibiaí & Miravânia & Santa Fé de Minas \\
Botumirim & Ibiracatu & Montalvânia & Santo Antônio do Retiro \\
Brasília de Minas & Icaraí de Minas & Monte Azul & São Francisco \\
Buritizeiro & Indaiabira & Montes Claros & São João da Lagoa \\
\hline
\end{tabular}

(Continua)

Quadro 1: Municípios Integrantes da Região Intermediária do Norte de Minas.

(Continuação)

\begin{tabular}{llll}
\hline & \multicolumn{3}{c}{ Municípios } \\
\hline Campo Azul & Itacambira & Montezuma & São João da Ponte \\
Capitão Enéas & Itacarambi & Ninheira & São João das Missões
\end{tabular}




\begin{tabular}{llll} 
Catuti & Jaíba & Nova Porteirinha & São João do Pacuí \\
Chapada Gaúcha & Janaúba & Novorizonte & São João do Paraíso \\
Claro dos Poções & Januária & Olhos D' Água & São Romão \\
Cônego Marinho & Japonvar & Padre Carvalho & Serranópolis de Minas \\
Coração de Jesus & Jequitaí & Pai Pedro & Taiobeiras \\
Cristália & Josenópolis & Patis & Ubaí \\
Curral de Dentro & Juramento & Pedras de Maria da Cruz & Urucuia \\
Divisa Alegre & Juvenília & Pintópolis & Vargem Grande do Rio Pardo \\
Engenheiro Navarro & Lagoa dos Patos & Pirapora & Várzea da Palma \\
Espinosa & Lassance & Ponto Chique & Varzelândia \\
Francisco Dumont & Lontra & Porteirinha & Verdelândia \\
Francisco Sá & Luislândia & Riachinho & \\
Fruta de Leite & Mamonas & Riacho dos Machados & \\
Gameleiras & Manga & Rio Pardo de Minas & \\
\hline & & Fonte: IBGE, 2020. &
\end{tabular}

Sendo, a região escolhida para este estudo, uma das maiores regiões geográficas intermediárias do estado de Minas Gerais, trabalhos que busquem empiricamente verificar e analisar o comportamento conjuntural desses municípios são primordiais para mensuração da qualidade de vidas desses munícipes, por exemplo.

\section{Tratamento das Variáveis e Construção do Índice}

Para a construção do Índice Relativo de Qualidade de Vida - IRQV para as regiões geográficas do conglomerado norte mineiro foram utilizadas técnicas relativas à análise fatorial. "A analise fatorial possui propriedades importantes, sendo que uma delas refere-se à ortogonalidade dos fatores" (CARDOSO et al., 2015, p. 352).

$\mathrm{Na}$ melhoria interpretativa dos fatores, este método proporciona o modelo que dispõe de uma melhor análise e composição. Para este estudo, foi utilizado o método Varimax de rotação ortogonal que busca minimizar o número de variáveis relacionadas diretamente com cada fator escolhido na estrutura do estudo.

\section{Análise Fatorial}

Para realizar o cálculo do IRQV dos municípios componentes da região geográfica intermediária do Norte de Minas Gerais/MG, o modelo disposto por Cardoso et al. (2015, p. 351) é exemplificado da seguinte forma: 
$X_{i=} a_{i j} f_{i}+\varepsilon_{i}$

sendo que $X_{i}=\left(X_{1}, X_{2}, X_{3}, \ldots, X_{p}\right)^{t}$ é um vetor transposto de variáveis observáveis aleatórias; $f_{i}=\left(f_{1}, f_{2}, f_{3}, \ldots, f_{r}\right)^{t}$ é um vetor transposto $(r<p)$ de variáveis não observáveis ou de fatores; $a_{i j}$ é uma matriz $(p x r)$ de coeficientes fixos denominados cargas fatoriais; e $\varepsilon_{i}=$ $\left(\varepsilon_{1}, \varepsilon_{2}, \varepsilon_{3}, \ldots, \varepsilon_{p}\right)^{t}$ é um vetor transposto de erros aleatorizados.

"A análise fatorial possui propriedades importantes, sendo que uma delas refere-se à ortogonalidade dos fatores. Nem sempre a estrutura inicial das estimativas das cargas fatoriais é definitiva". (CARDOSO et a.l, 2015, p. 352). Neste trabalho foi utilizado o método Varimax de rotação para minimizar o número de variáveis que possuem relação forte com cada um dos fatores.

Na construção do IRQV a estimativa foi relacionada com a proporção de explicação que a metodologia apresentou sobre a prerrogativa explicativa que cada fator extraído após a rotação apresentou no sistema. "Por definição, o escore fatorial irá situar cada observação no espaço dos fatores comuns, de modo que, para cada fator $f_{i}$, o i-ésimo extraído é definido por $F_{i}[\ldots]$... (CARDOSO et al., 2015, p. 352).

\section{Índice Relativo de Qualidade de Vida (IRQV)}

Após ser realizada a extração dos fatores, a mensuração do índice relativo de qualidade de vida - IRQV foi realizada em duas etapas. A primeira etapa constitui um IRQV que tem como base de referência o melhor IQ $V_{i}$ dos municípios mineiros da região geográfica intermediária escolhida para este estudo. A definição do IQ $V_{i}$ foi mensurada pela seguinte equação apresentada por Cardoso et al. (2015, p. 354):

$\mathrm{IQ} V_{i}=\sum_{j=1}^{p} \frac{\sigma_{j}^{2}}{\sum \sigma_{j}^{2}} F_{i j}^{*}$

em que o IQ $V_{i}$ é o índice de qualidade de vida do i-ésimo município mineiro da região intermediária escolhida para estudo; $p$ é o número de fatores utilizados para realizar a análise, $F_{i j}^{*}$ é o j-ésimo escore da análise fatorial do i-ésimo município mineiro; $\sum \sigma_{j}^{2}$ é o somatório das variâncias explicadas pelo $p$ fatores extraídos; e $\frac{\sigma_{j}^{2}}{\sum \sigma_{j}^{2}}$ indica a participação relativa do fator $j$ na devida explicação da variância total apresentada pelos $p$ fatores extraídos. 
Há a necessidade de que os escores apresentados pelos municípios apresentem uma distribuição simétrica em média zero. "Assim, metade deles apresentará sinais negativos e, a outra metade, sinais positivos, de modo que os municípios com menores índices parciais de qualidade de vida apresentarão escores fatoriais negativos" (CARDOSO et al., 2015, p. 355).

Como forma de evitar que os altos escores negativos elevem os resultados dos índices há a necessidade de transformação associada na seguinte fórmula:

$F_{i j}=\frac{\left(F_{i j}-F_{i}^{\text {min }}\right)}{F_{i}^{\text {max }}-F_{i}^{\text {min }}}$,

em que $F_{i}^{\text {min }}$ e $F_{i}^{\text {máx }}$ são os valores máximos e mínimos observados para o j-ésimo escore fatorial interligado ao i-ésimo município da região geográfica intermediária do Norte de Minas Gerais. Após obter o IQ $V_{i}$ foi executado o cálculo do IRQ $V_{i}$ realizando com base na equação apresentada por Cardoso et al. (2015, p. 355):

$\operatorname{IRQ} V_{i}=\left[\left(\frac{I Q V_{i}}{I Q V_{i}^{M A X}}\right) * 100\right]$

onde $\mathrm{IQ} V_{i}$ é o valor do índice de qualidade de vida do i-ésimo município da região intermediária selecionada para análise e o IQ $V_{i}^{\text {máx }}$ o maior valor obtido para o IQ $V_{i}$ da $i$ ésima unidade espacial melhor posicionada no ranking.

"Quando maior for o resultado obtido relativamente ao maior do IRQ $V_{i}$, melhor será a situação do município em termos de qualidade de vida" (CARDOSO, et al. 2015, p. 355). Desta forma é possível não só realizar o posicionamento dos níveis de qualidade de vida, mas também observar quais municípios da região intermediária escolhida apresentam um maior ou menor desenvolvimento do IQ $V_{i}$, além de permitir que os municípios sejam comparados entre si no quesito similaridades.

\section{Base de Dados e Seleção das Variáveis}

Para construção do Índice Relativo de Qualidade de Vida dos municípios componentes da região intermediária do Norte de Minas Gerais/MG - IRQV, foram selecionadas 17 indicadores obtidos no último censo realizado pelo IBGE em 2010 relacionados às áreas da saúde, renda, educação, habitação, vulnerabilidade, cultura, esporte e lazer. 
A escolha das variáveis se deu pelas dimensões primárias utilizadas para cálculo do $\mathrm{IDH}^{4}$. Porém, “devido à complexidade inerente ao conceito de desenvolvimento, [...], decidiuse pro agregar outras variáveis que contemplem aspectos associados à infraestrutura urbana, à vulnerabilidade e à cultura, esporte e lazer” (CARDOSO et al., 2015, p. 356). Para a construção do IRQV foram utilizados os seguintes indicadores:

a) Saúde:

- IX1 - proporção de óbitos por causas mal definidas sem assistência médica;

- IX2 - proporção de internações hospitalares por condições sensíveis à atenção primária;

- IX3 - proporção das internações de média complexidade de pacientes do SUS encaminhados para outra microrregião; e

- IX4 - esperança de vida ao nascer.

b) Renda:

- IX5 - renda per capita;

- IX6 - empregados do setor formal; e

- IX7 - taxa de emprego no setor formal.

c) Educação:

- IX8 - proporção de pessoas de 15 anos ou mais de idade com ensino fundamental completo; e

- IX9 - taxa de atendimento escolar das crianças de 0 a 3 anos de idade.

d) Habitação:

- IX10 - percentual da população em domicílios com energia elétrica;

- IX11 - percentual da população em domicílios com banheiro e água encanada;

- IX12 - percentual da população em domicílios com coleta de lixo (direta e indireta); e

- IX13 - número de domicílios urbanos próprios com adensamento excessivo.

e) Vulnerabilidade:

- IX14 - razão entre a renda média dos $20 \%$ mais ricos e os $40 \%$ mais pobres;

- IX15 - percentual de vulnerabilidade e dependentes de idosos, no total de vulneráveis que coabitam com idosos; e

- IX16 - percentual de extremamente pobres.

f) Cultura, esporte e lazer:

${ }^{4}$ O Índice de Desenvolvimento Humano busca medir as condições de renda, saúde e educação sobre o qual estão submetidas às populações de um determinado país. Informação disponível em <https://www.br.undp.org/content/brazil/pt/home/idh0/conceitos/o-que-e-o-idh.html $>$. Acesso em 27/01/2020. 
- IX17 - gasto per capita com difusão cultural.

Os indicadores selecionados foram transformados em números-índice, tendo como base 100 o maior valor obtido em cada indicador para cada município, sendo que, a base de dados secundária utilizada foi obtida pelo Atlas de Desenvolvimento Humano no Brasil (PNUD, 2019) e do Censo Demográfico Brasileiro de 2010 realizado pelo IBGE.

\section{Análise dos Dados e Resultados}

A partir da realização da análise fatorial dos indicadores referentes à qualidade de vida foi possível obter o resultado dos fatores e o percentual de explicação das cargas fatoriais. A extração foi feita sobre seis (6) fatores que em conjunto apresentaram um grau de explicação em torno de $74 \%$ da variância total contida nos dezessete (17) indicadores selecionados para este estudo. Os dados obtidos em cada fator na rodagem dos dados são apresentados a seguir:

Tabela 1: Resultado após a rotação dos dados pelo método Varimax.

\begin{tabular}{c|c|c|c}
\hline Fator & Autovalor & Variância Explicada pelo fator (\%) & Variância Acumulada (\%) \\
\hline 1 & 5,24 & 20,32 & 20,32 \\
2 & 1,95 & 19,46 & 39,78 \\
3 & 1,66 & 9,33 & 49,11 \\
4 & 1,38 & 8,75 & 57,86 \\
5 & 1,29 & 8,16 & 66,02 \\
6 & 1,00 & 7,61 & 73,63 \\
\hline
\end{tabular}

Fonte: Elaborado pela Autora.

Como explica Cardoso et al. (2015, p. 358) a metodologia aplicada para a realização da análise fatorial padece de um padrão que determine quantos fatores devem ser extraídos, porém, neste estudo, o condicionamento de extração até o sexto fator foi retratada pelo software econométrico Stata 14.0, sendo este o último resultado apresentado que proporciona um auto valor de explicação juntamente com a proporção de explanação apresentada pelas variáveis aplicáveis ao modelo estrutural dos dados.

Tabela 2: Cargas fatoriais após rotação ortogonal pelo método Varimax e comunalidades

\begin{tabular}{l|c|c|c|c|c|c|c}
\hline Indicadores & Fator 1 & Fator 2 & Fator 3 & Fator 4 & Fator 5 & Fator 6 & Comunalidades \\
\hline IX1 & 0,06 & $-0,08$ & 0,02 & $\mathbf{- 0 , 7 8}$ & 0,10 & $-0,11$ & 0,35 \\
IX2 & $-0,09$ & 0,01 & $-0,33$ & $-0,05$ & $\mathbf{0 , 7 4}$ & 0,09 & 0,32 \\
IX3 & $-0,09$ & $-0,36$ & $-0,32$ & 0,17 & $\mathbf{- 0 , 6 0}$ & $-0,19$ & 0,34 \\
IX4 & $\mathbf{0 , 6 3}$ & 0,17 & $-0,09$ & 0,28 & $-0,18$ & 0,00 & 0,45 \\
IX5 & $\mathbf{0 , 6 5}$ & 0,48 & 0,00 & 0,21 & $-0,04$ & 0,36 & 0,17 \\
IX6 & $\mathbf{0 , 9 4}$ & 0,09 & 0,01 & $-0,08$ & 0,02 & 0,06 & 0,09 \\
IX7 & 0,36 & $\mathbf{0 , 6 7}$ & $-0,04$ & $-0,18$ & $-0,13$ & 0,32 & 0,27
\end{tabular}




\begin{tabular}{l|r|r|r|r|r|r|r} 
IX8 & $\mathbf{0 , 6 2}$ & 0,43 & $-0,03$ & 0,29 & $-0,18$ & 0,12 & 0,29 \\
IX9 & 0,09 & $-0,01$ & $\mathbf{0 , 8 6}$ & 0,07 & 0,04 & $-0,14$ & 0,23 \\
IX10 & 0,14 & 0,46 & 0,16 & $\mathbf{0 , 5 6}$ & 0,19 & 0,00 & 0,39 \\
IX11 & 0,32 & $\mathbf{0 , 6 8}$ & $-0,10$ & 0,31 & 0,08 & 0,10 & 0,31 \\
IX12 & 0,17 & 0,02 & 0,03 & 0,08 & 0,13 & $\mathbf{0 , 8 4}$ & 0,24 \\
IX13 & $\mathbf{0 , 9 5}$ & 0,08 & $-0,02$ & $-0,05$ & 0,02 & 0,05 & 0,09 \\
IX14 & 0,17 & $\mathbf{- 0 , 7 6}$ & $-0,04$ & 0,06 & $-0,11$ & 0,39 & 0,24 \\
IX15 & $-0,10$ & $-0,42$ & 0,20 & 0,37 & $\mathbf{0 , 5 4}$ & $-0,09$ & 0,34 \\
IX16 & 0,24 & $\mathbf{- 0 , 9 1}$ & 0,04 & $-0,15$ & 0,01 & $-0,04$ & 0,09 \\
IX17 & 0,24 & $-0,08$ & $\mathbf{0 , 7 4}$ & $-0,06$ & $-0,15$ & 0,30 & 0,27 \\
\hline
\end{tabular}

Fonte: Elaborado pela Autora.

O fator 1 apresenta um maior grau de variância $(20,32 \%)$ entre os seis fatores apresentados está positivamente associado aos indicadores IX4 (esperança de vida ao nascer), IX5 (renda per capita), IX6 (empregados do setor formal), IX8 (proporção de pessoas de 15 anos ou mais de idade com ensino fundamental completo) e IX13 (número de domicílios urbanos próprios com adensamento excessivo). Desta forma, quanto mais elevado é a representação deste, maior o nível de qualidade de vida do município. $O$ fator 1 não apresentou variância correlacional negativa significativa. Estes resultados denotam que os indicadores apresentaram uma maior correlação entre as variáveis relacionadas à educação, remuneração e emprego, o fator 1 foi renomeado passando a ser definido como "acesso ao arcabouço social".

O fator 2 apresentou a segunda maior correlação $(19,46 \%)$ com as variáveis escolhidas na sistemática possui uma correlação positiva com a variável IX7 (taxa de emprego no setor formal) e IX11 (percentual da população em domicílios com banheiro e água encanada). A correlação negativa foi relacionada às variáveis IX14 (razão entre a renda média dos 20\% mais ricos e os $40 \%$ mais pobres) e IX16 (percentual de extremamente pobres). Como as variáveis positivas estão ligadas aos aspectos de renda/habitação e as variáveis negativas estão ligadas as condições de vulnerabilidade, o fator 2 foi renomeado para "condição habitacional e desigualdade de renda".

O fator 3 apresenta 9,33\% de explicação conjuntural das variáveis escolhidas para este estudo, sendo que a variável IX9 (taxa de atendimento escolar das crianças de 0 a 3 anos de idade) apresentou resultado positivo e a variável IX17 (gasto per capita com difusão cultural) apresentou resultado negativo. Assim sendo, foi renomeada para "difusão cultural na primeira infância".

O fator 4 apresenta um grau de explicação das variáveis de $8,75 \%$ tendo a variável IX10 (percentual da população em domicílios com energia elétrica) resultado positivo e a 
variável IX1 (proporção de óbitos por causas mal definidas sem assistência médica) resultado negativo. Por tanto, o fator 4 foi definido como "acesso a serviços ou atividades essenciais".

O fator 5 apresenta um grau de explicação de $8,16 \%$, sendo que as variáveis IX2 (proporção de internações hospitalares por condições sensíveis à atenção primária) e IX15 (percentual de vulnerabilidade e dependentes de idosos, no total de vulneráveis que coabitam com idosos) resultados positivos e a variável IX3 (proporção das internações de media complexidade de pacientes do SUS encaminhados para outra microrregião) resultado negativo. A nova nomenclatura adotada neste fator foi "acesso à saúde pública".

Por fim, o fator 6 que apresenta percentual explicativo de 7,61\% da variância, na apresenta correlações negativas significantes entre as variáveis e apresentou positivamente correlação na variável IX12 (percentual da população em domicílios com coleta de lixo, direta ou indireta) tendo esta variável denomina de "coleta de lixo".

Os dados foram relacionados nos mapas 2 a 7 que representam a orientação espacial dos fatores. A partir disto, foram criadas as legendas categorias para melhor visualização dos resultados resultantes dos fatores elencadas como muito baixo, baixo, médio, alto e muito alto. Foram colocados também nas legendas os municípios não analisados neste trabalho por estarem fora da área de abrangência da região intermediária norte mineira.

Mapa 2: Acesso ao Arcabouço Social.

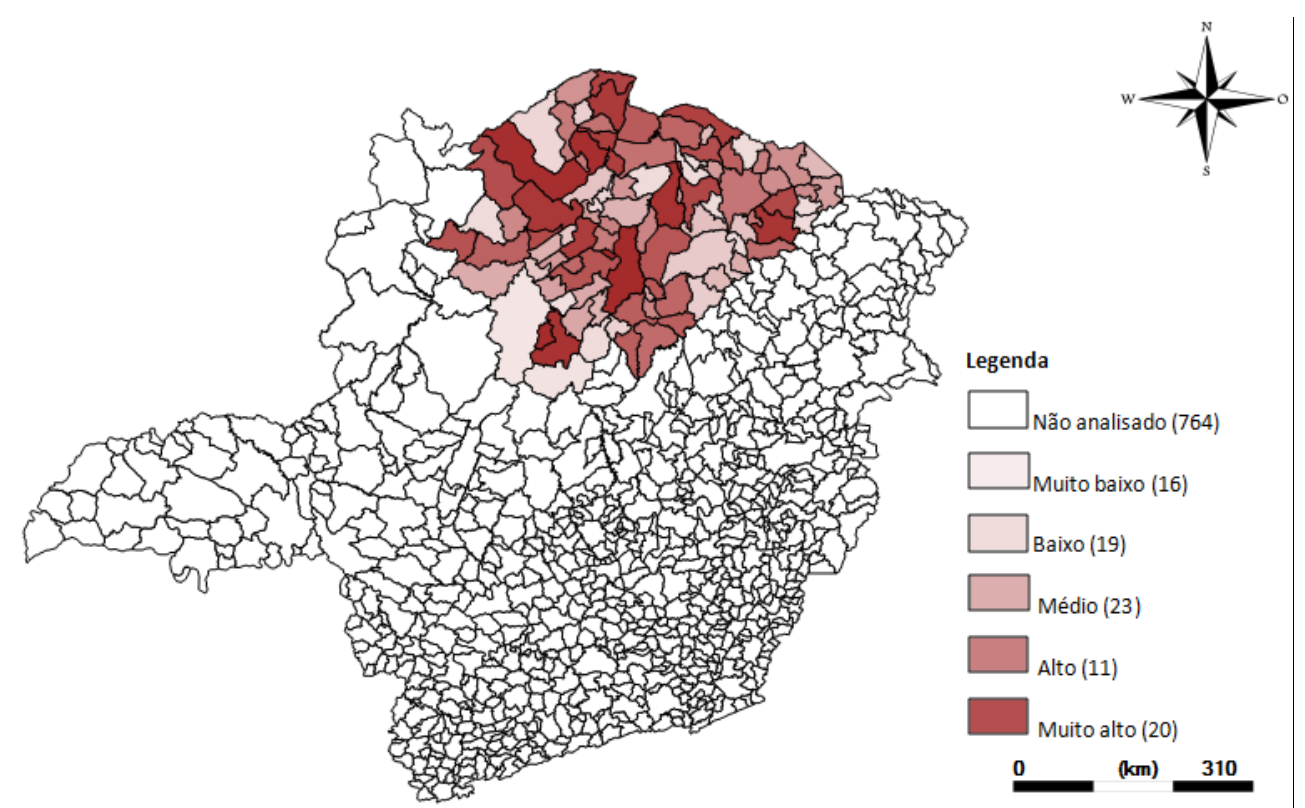

Fonte: Elaborado pela autora. Software IpeaGEO.

A analise resulta em condicionantes relativamente favoráveis de acesso pela população nesta área, fazendo com que as colocações regionais não apresentem tanta discrepância de 
resultados. Desta forma, comparando os municípios da região estudada no mapa há a evidenciação de que as diferenças deste quesito não apresentam grandes lacunas direcionais entre as cidades.

Os resultados apresentados aqui podem estar relacionados diretamente com os índices educacionais que a região norte mineira apresenta mesmo esta ser polo de universidades e faculdades, e de apresentar uma preocupação com o desenvolvimento educacional no desenvolvimento do estado.

Neste caso, houve uma demonstração de correlação entre o nível de educação influência diretamente no valor da renda e emprego. A representatividade Norte Mineira apresenta alguns pontos muito altos e altos, porém a distribuição na consegue ser completa apresentando pontos em que a variável cai consideravelmente.

Mapa 3: Condição Habitacional e Desigualdade de Renda.

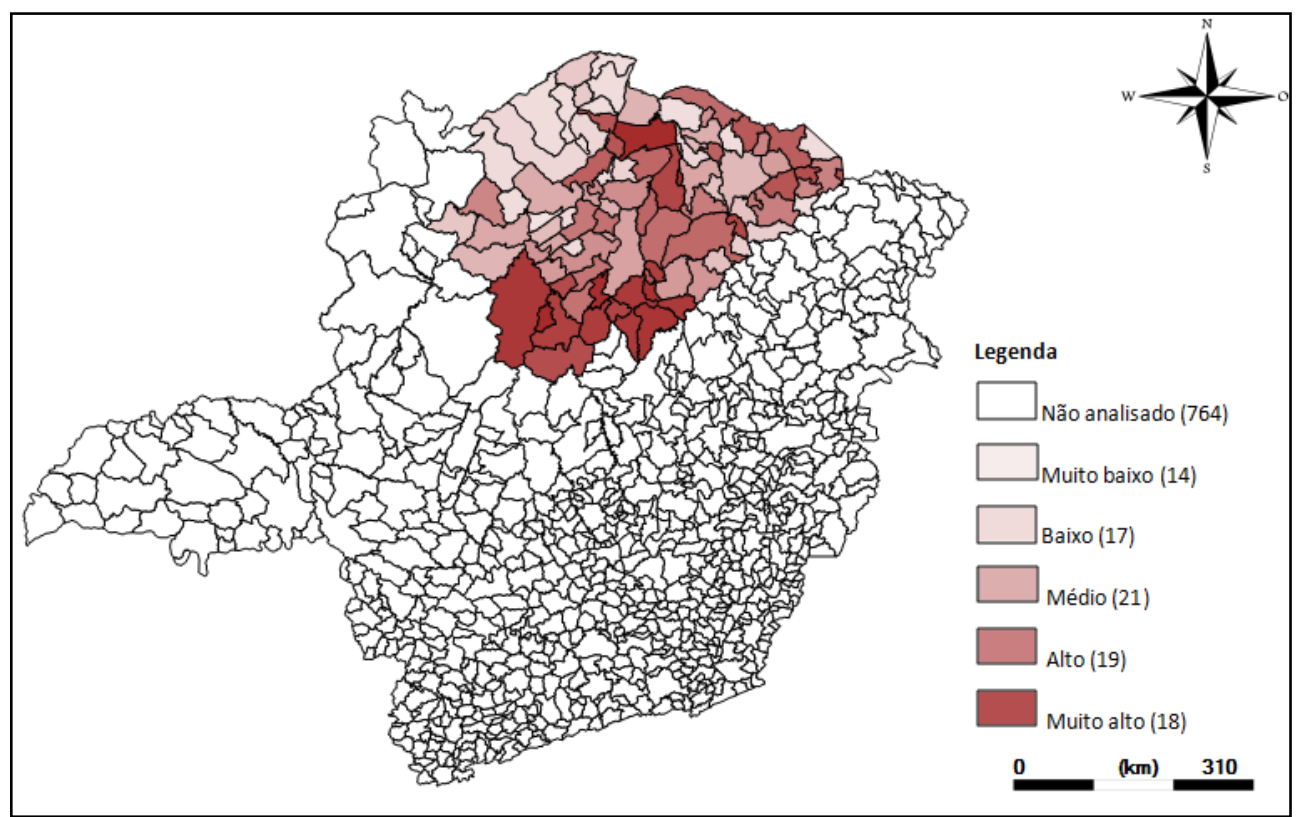

Fonte: Elaborado pela autora. Software IpeaGEO.

Nesta análise os condicionantes divergem do anterior, apresentando áreas com colorações mais escuras nas cidades do norte da região intermediária do Norte de Minas e uma coloração mais clara nas regiões próximas ao Sul do estado da Bahia, por exemplo. Isso denota as lacunas apresentadas pelas variáveis nas cidades estudadas e suas disparidades com outras regiões. 
Mapa 4: Difusão Cultural na Primeira Infância.

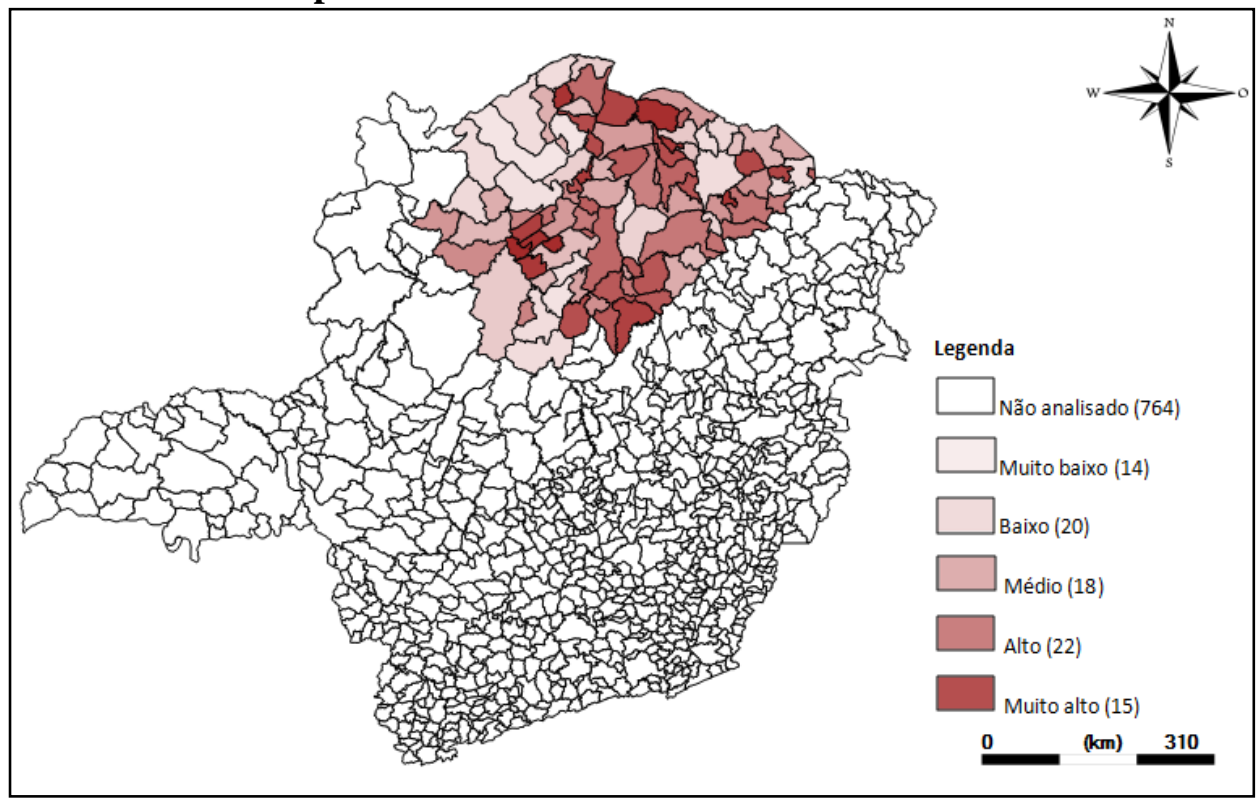

Fonte: Elaborado pela autora. Software IpeaGEO.

Relaciona as dimensões entre os gastos realizados com lazer para o público infantil da região do conglomerado norte mineiro. $\mathrm{O}$ mapa 4 demonstra uma proporção de gastos com similaridades em muitas cidades realizando o pensamento de representatividade consideravelmente relevante para com os munícipes.

Mapa 5: Acesso a Serviços ou Atividades Essenciais.

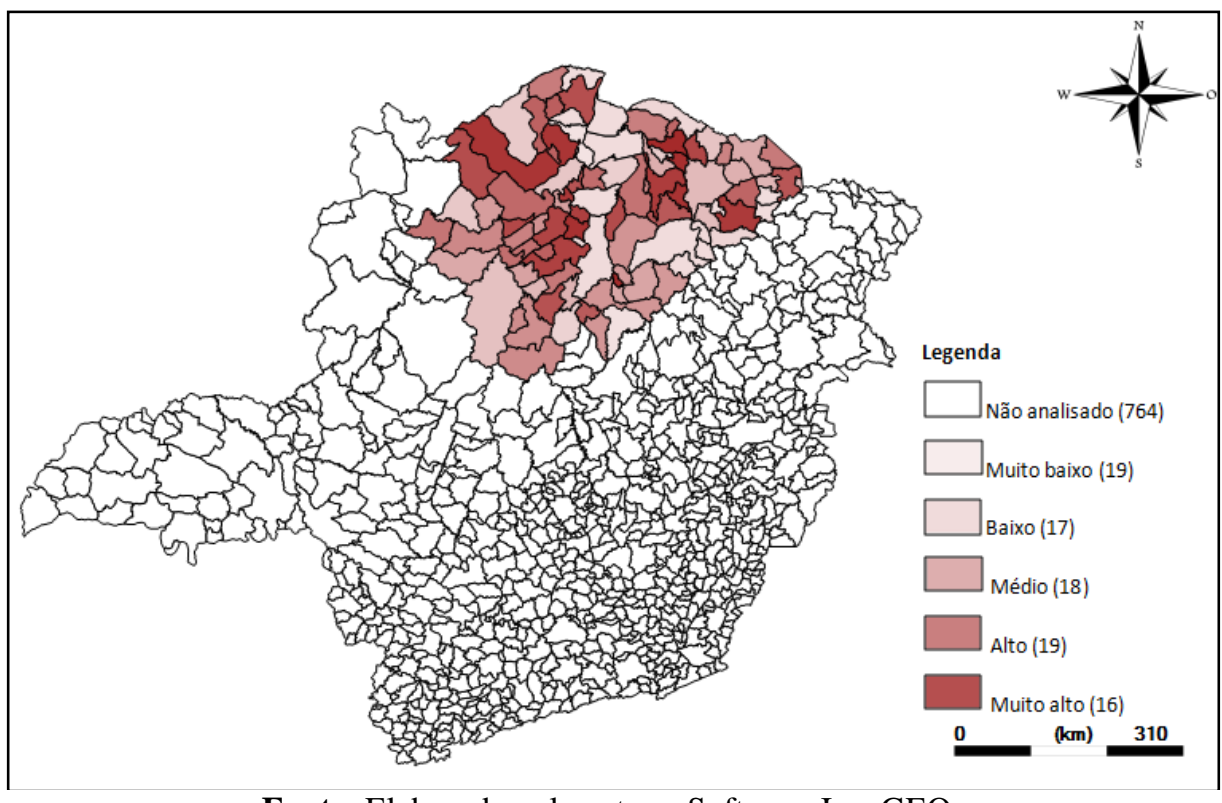

Fonte: Elaborado pela autora. Software IpeaGEO. 
Ao analisarmos os dados na estrutura revelada pelo mapa 5, revela-se que a conjuntura desta variável possuiu um número razoável de municípios que garantem acesso aos serviços essenciais para seus munícipes, assim como no mapa 6 apresenta o acesso à saúde pelos munícipes demonstrando observância de garantia com uma boa representação na região.

O que ocorre nesta variável dimensiona pequenas escalas de municípios que conseguem realizar a redistribuição satisfatória de serviços públicos essenciais e vizinhos que acabam não realizando a redistribuição da mesma maneira. A proporção apresentadas de municípios muito baixo (19) e baixo (17) foi maior que a apresentada entre os altos (19) e os muito altos (16).

O mapa 6 representa o acesso à saúde apresenta os maiores índices no entorno com o município da Bahia e em pequenos conglomerados no sul da região intermediária do Norte de Minas, representando que os serviços de saúde ainda representam um declínio grande se comparamos com outras regiões do estado. Desta forma, a representação evidência a deficiência na execução e na entrega de saúde aos munícipes residentes na região intermediária norte mineira.

Mapa 6: Acesso à Saúde.

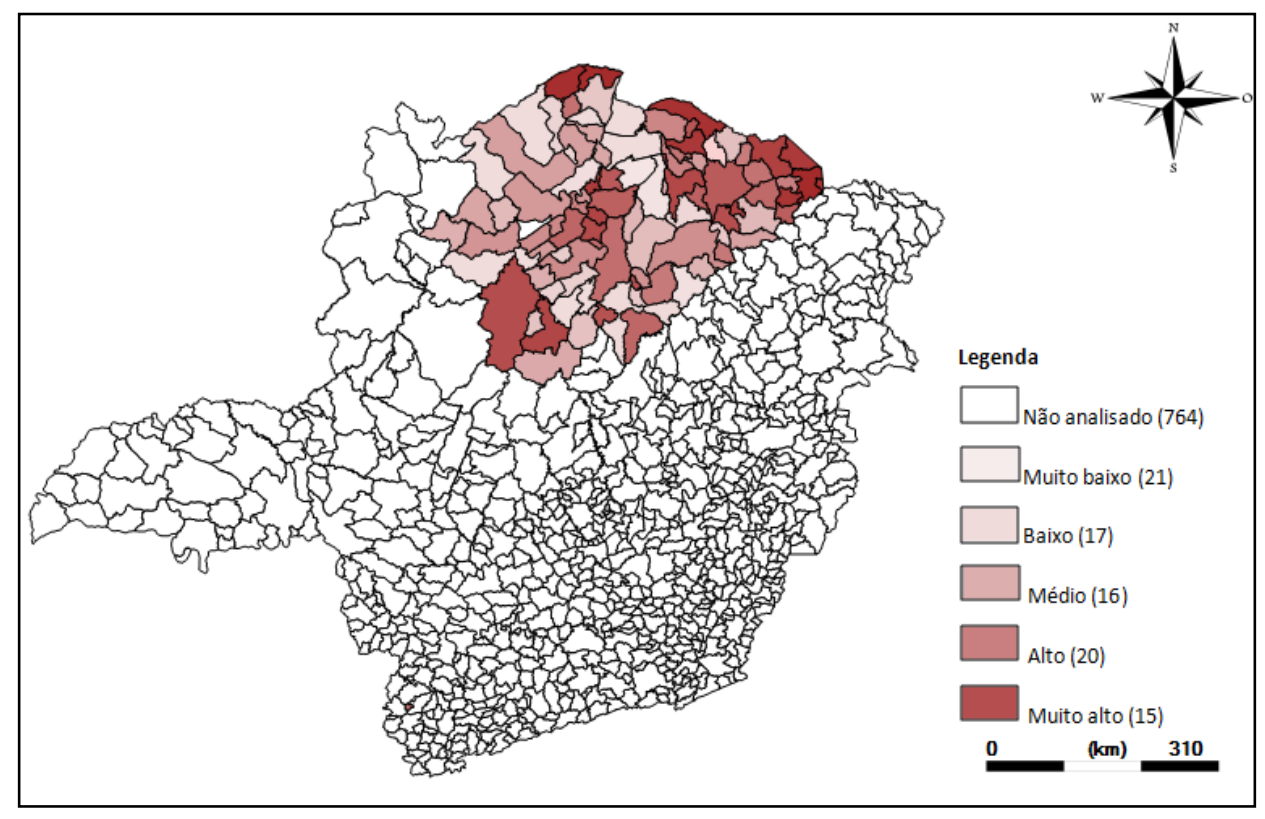

Fonte: Elaborado pela autora. Software IpeaGEO. 
Mapa 7: Coleta de Lixo.

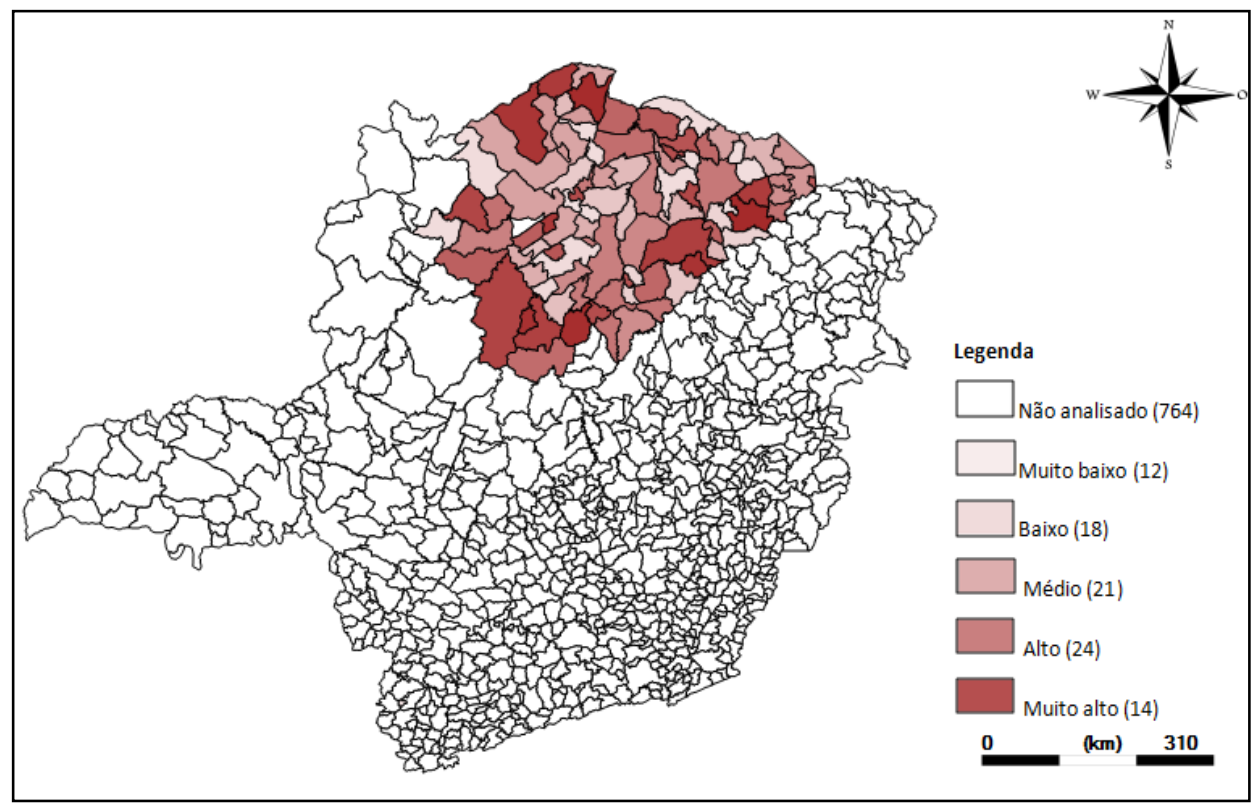

Fonte: Elaborado pela autora. Software IpeaGEO.

A representação desta variável denota algo parecido com a demonstrada no mapa 6, assim como nas variáveis relacionadas à saúde, a região Norte Mineira tem apresentado indicativos de deficiências na saúde, acontecendo o mesmo com aspectos relacionados à garantia de serviços públicos essenciais, como o de coleta seletiva de lixo, além de outras garantias. Os mapas apresentam conglomerados denominando avanços, porém há a existência de regiões deficitárias destes serviços essenciais para a população e sua saúde.

O estudo realizado por Cardoso et al. (2015, p. 363) para todos os municípios do estado de Minas Gerais demonstra que o IRQV maior reside nas regiões do Sudoeste, triangulo, Oeste e Centro-Sul de Minas. Há a representatividade maior do índice nestas localizações, que se explica principalmente pelo alto nível de industrialização dessas regiões, por exemplo. Para o Norte de Minas Gerais o estudo demonstra uma deficiência em comparação com as outras regiões mais industrializadas devido ao seu desenvolvimento tardio e suas atividades centrais ligadas a um menor valor agregado na economia, por exemplo, serviços.

Após análise de cada uma das variáveis apresentadas, a verificação do IRQV foi realizada para o ano de 2010 apresentando as características das diversificações dos municípios da região intermediária do Norte de Minas. Cardoso et al. (2015, p. 363) realizou a análise do IRQV para o estado de Minas Gerais constatando que as regiões Sudeste, Triângulo Mineiro, Oeste e Centro-Sul apresentaram o maior IRQV por apresentar um maior índice de industrialização sobre as demais regiões. Na mesma pesquisa, o Norte de Minas 
Gerais apresentou índices que, em sua classificação apresentava poucos municípios com médios e altos índices de IRQV.

Mapa 8: Índice Relativo de Qualidade de Vida para Todos os Municípios Mineiros.

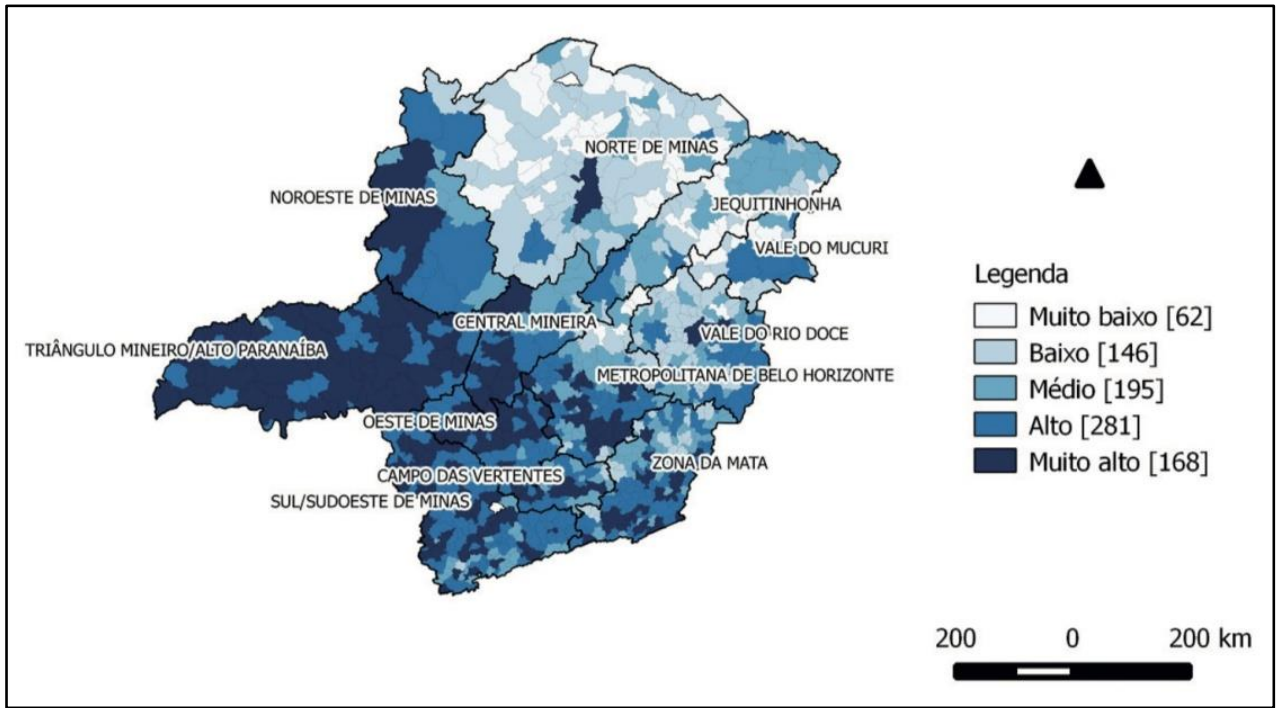

Fonte: CARDOSO et al., 2015, p. 363.

O mapa 8 demonstra que dos 89 (oitenta e nove) municípios que compõem a região intermediária do conglomerado norte mineiro, apenas município de Montes Claros apresenta um IRQV muito alto, sendo que os demais municípios deste conglomerado apresentaram variâncias maiores entre muito baixo e baixo. O mapa 9 demonstra a análise apenas com a região intermediária do Norte de Minas efetivando os seguintes resultados:

Mapa 9: IRQV - Norte de Minas.

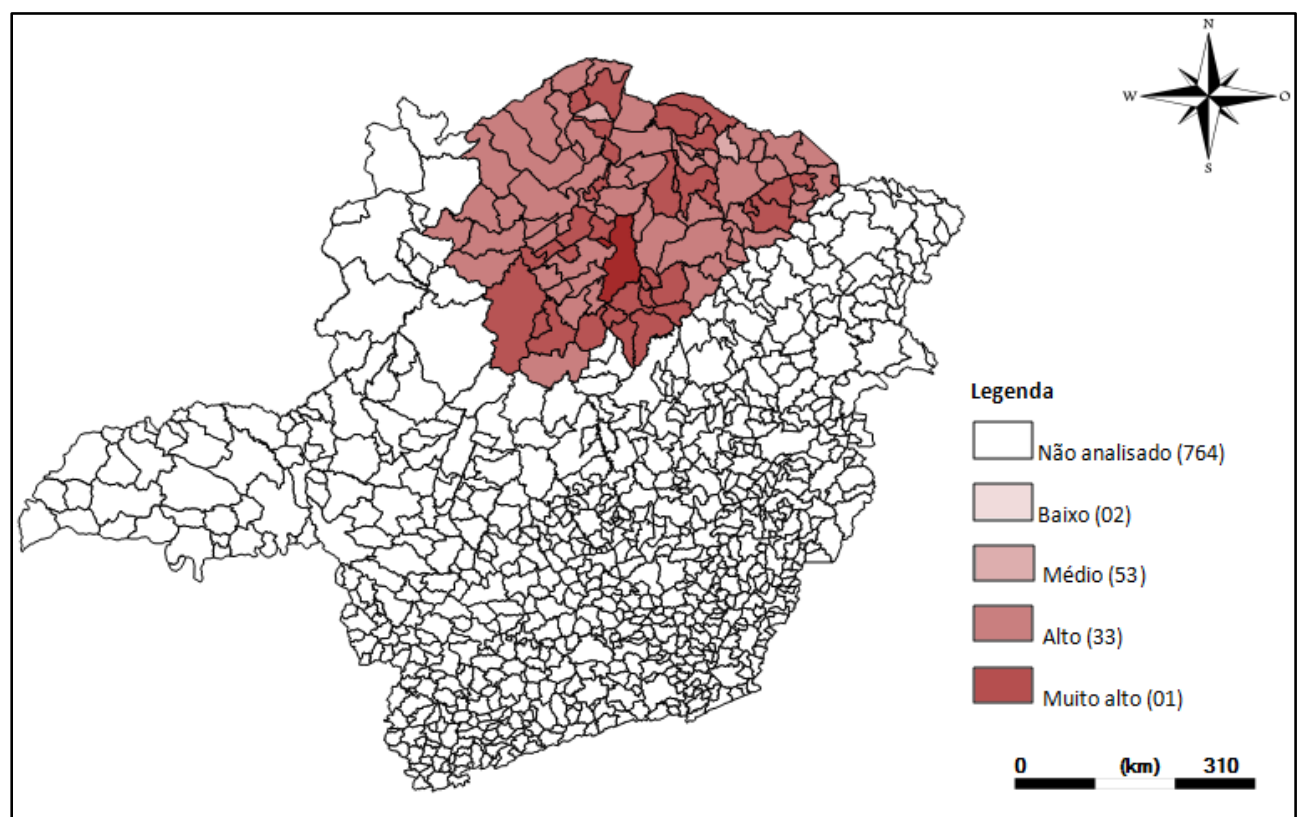

Fonte: Elaborado pela autora. Software IpeaGEO. 
O mapa 9 demonstra que quando realizamos a comparação da região Norte Mineira isolado das demais terá resultados diferentes do que a comparação entre as todas as regiões no estado em conjunto. Nesta análise, nenhum município demonstrou resultados muito baixos assim como apenas o município de Montes Claros apresentou um IRQV muito alto, confirmando o resultado apresentado no mapa 8 .

A tabela 3 demonstra os dez municípios que apresentaram o maior e o menor IRQV na região geográfica intermediária do Norte de Minas Gerais. O município de Montes Claros apresentou a melhor colocação em relação as demais cidades, ou seja, o melhor índice apresentado na região intermediária foi de 100\%. Em contrapartida, o município de São João das Missões apresentou o pior resultado ficando na última colocação com 38,60\% na região estudada. Desta maneira, podemos confirmar que os indicativos de qualidade de vida em Montes Claros representam mais que o dobro que no município de São João das Missões.

Tabela 3: Índice Relativo à Qualidade de Vida na Região Intrmediária do Norte de Minas Gerais. $(\mathrm{Em} \%)$

\begin{tabular}{l|r|l|r}
\hline Dez municípios com maior IRQV & IRQV & Dez municípios com menor IRQV & IRQV \\
\hline Montes Claros & 100 & Cristália & 50,87 \\
Pirapora & 77,34 & Pedras de Maria da Cruz & 50,84 \\
Salinas & 70,02 & Cônego Marinho & 50,66 \\
Taiobeiras & 67,33 & Jequitaí & 50,12 \\
Várzea da Palma & 67,03 & Josenópolis & 49,59 \\
Janaúba & 66,54 & Santa Cruz de Salinas & 48,37 \\
Engenheiro Navarro & 64,85 & Chapada Gaúcha & 43,53 \\
Olhos D’Água & 64,59 & Bonito de Minas & 40,65 \\
Ponto Chique & 64,55 & Santo Antônio do Retiro & 39,27 \\
São João do Pacuí & 64,44 & São João das Missões & 38,60 \\
\hline
\end{tabular}

Fonte: Elaborado pela Autora.

Apesar de haver uma lacuna grande entre o primeiro colocado (Montes Claros) e o último (São João das Missões), as demais cidades localizadas no campo deste estudo apresentaram, em sua grande maioria, valores próximos na escala de relatividade, principalmente se compararmos ao estudo similar realizado por Cardoso et al. (2015, p. 364). Isso porque a representatividade foi comparada em relação à totalidade dos municípios mineiros, sendo que as regiões mineiras se desenvolveram em períodos e de forma diferente.

Deste modo, consegue-se mensurar que a relatividade da região geográfica intermediária do Norte de Minas Gerais apresenta um alto grau de equidade nos municípios componentes desta pesquisa. Há também a necessidade de se atentar as verificações de que os dados são de dez anos atrás (Censo 2010), portanto deve-se continuar acompanhando o 
crescimento e o aperfeiçoamento da região intermediária norte mineira para melhor compreensão das necessidades e de crescimento produtivo da região.

\section{Considerações Finais}

O presente estudo elaborou o Índice Relativo de Qualidade de Vida da Região Geográfica Intermediária do Norte de Minas Gerais/MG apontando quais os municípios do conglomerado que apresentava os melhores índices. Para tanto, foi utilizada a analise fatorial através do software Stata 14.0 para cálculo e representação das variáveis escolhidas para este.

Os resultados apresentados pelos municípios estudados apresentam traços heterogêneos dos municípios ao mesmo tempo em que representam similaridades entre eles. Aspectos interligados a saúde e educação, por exemplo, apresentaram similaridades regionais que afastam estas regiões das estruturas de desenvolvimentos já conquistadas por outros, como, por exemplo, o Triângulo mineiro e a região Metropolitana de Belo Horizonte.

O índice constructo apresentou que o município de Montes Claros detinha de uma maior relação de qualidade do que os municípios da região geográfica intermediária do Norte de Minas, confirmando os resultados que Cardoso et al. (2015, p. 363) também já havia conseguido demonstrar em seu estudo. Infelizmente, sua pesquisa reafirma que da região geográfica norte mineira, apenas Montes Claros conseguiu este posicionamento.

Quando analisado somente o conglomerado em relação a ele mesmo, o índice de qualidade de vida demonstra uma alta frequência de municípios apresentando variáveis de média (53) de baixa (33), já que vários aspectos foram integrados e concatenados a fim de demonstrar como as variáveis se compõem nesta região. Além disso, vale salientar que apenas dois (2) municípios norte mineiros apresentaram um índice de qualidade de vida baixo demonstrando há um desenvolvimento regional do conglomerado ou podendo demonstrar também que há um grau de estagnação similar nos municípios da presente região.

Vale ressaltar que os dados utilizados nesta pesquisa são do Censo de 2010, portanto vários aspectos podem ter sido modificados nestas localidades, o que ressalva a necessidade de estudos futuros com dados mais recentes, por exemplo.

Com isso, há necessidade de salientar que o desenvolvimento tardio da região geográfica intermediária do Norte de Minas Gerais/MG contribui para a disparidade dos aspectos econômicos e sociais apresentados, principalmente nos campos da saúde e educação, contribuindo para uma estrutura pobre e precária que estende a desqualificação desmerecida 
que a região estabelece, mas que merece uma maior atenção por parte do poder público, a fim ajudar a aumentar a qualidade desses municípios e de seus munícipes.

\section{Referências}

AGÊNCIA DE NOTÍCIAS IBGE. Disponível em: $<$ https://agenciadenoticias.ibge.gov.br/agencia-sala-de-imprensa/2013-agencia-denoticias/releases/10515-ibge-divulga-nova-divisao-territorial-com-foco-nas-articulacoesregionais>. Acesso em 02 de jan. 2020.

AVERBUG, André. Abertura e Integração Comercial Brasileira na Década de 90. In: GIAMBIAGI, Fabio; MOREIRA, Maurício Mesquita. A Economia Brasileira nos Anos 90. 1. ed. Rio de Janeiro: Banco Nacional de Desenvolvimento Econômico e Social, 1999. 488 p.

BRASIL, Constituição Federal de 1988. Disponível em: 〈http://www.planalto.gov.br/ccivil_03/constituicao/constituicao.htm>. Acesso em 15 de jul. 2019.

BRESSER-PERREIRA, Luiz Carlos. Crescimento e Desenvolvimento Econômico. Disponível em: $\quad$ https://www.researchgate.net/profile/Luiz_BresserPereira/publication/266495722_O_processo_historico_do_desenvolvimento_economico/links 157c75cb608ae9d64047e9979.pdf>. Acesso em: 21 de dez. 2019.

CARDOSO, Débora Freire. et al. Índice Relativo de Qualidade de Vida para os Municípios de Minas Gerais. In: Planejamento e Políticas Públicas. ppp, n. 45, jul/dez. 2015.

DINIZ, Alexandre Magno Alves; BATELLA, Wagner Barbosa. O Estado de Minas Gerais e suas Regiões: Um Resgate Histórico das Principais Propostas Oficiais de Regionalização. In: Sociedade e Natureza. Uberlândia, n. 17 (33): dez. 2005. 59-77 p.

FURTADO, Celso. Economia do desenvolvimento: curso ministrado na PUC-SP em 1975/Celso Furtado - Rio de Janeiro: Contraponto: Centro Internacional Celso Furtado, 2008.il. - (Arquivo Celso Furtado; v.2). 254 p.

INSTITUTO BRASILEIRO DE GEOGRAFIA E ESTATÍSTICA - IBGE. Divisão do Brasil em Mesorregiões e Microrregiões Geográficas. Volume 1. Rio de Janeiro, 1990. 135 p.

INSTITUTO BRASILEIRO DE GEOGRAFIA E ESTATÍSTICA - IBGE. Disponível em: <https://www.mg.gov.br/sites/default/files/paginas/arquivos/2016/ligminas_10_2_04_listame somicro.pdf $>$. Acesso em 02 de jan. 2020.

IpeaGEO. Versão 2.1. [S.1.]: IpeaGEO, 21 jan. 2020. Disponível em: <http://www.ipea.gov.br/ipeageo/download.html>. Acesso em 21 de dez. 2019.

PNUD - programa das Nações Unidas para o Desenvolvimento. Atlas de Desenvolvmento Humano no Brasil. Disponível em: 〈http://www.atlasbrasil.org.br/2013/pt/consulta/>. Acesso em: 21 de nov. 2019. 
Índice Relativo de Qualidade de Vida da Região Geográfica Intermediária do Norte de Minas Gerais/MG. Junia de Souza Silva

SCHUMPETER, Joseph Alois. A Teoria do Desenvolvimento Econômico. Uma Investigação sobre Lucros, Capital, Crédito, Juro e o Ciclo Econômico. São Paulo. Ed. Abril Cultural, 1982. 169 p.

STATA - Statistics/Data Analysis. StataCorp. Versão 14.0. [S.p].

UNDP - Programa das Nações Unidas para o Desenvolvimento Brasil. IDH - Índice de Desenvolvimento Humano. Disponível em: <https://www.br.undp.org/content/brazil/pt/home/idh0/conceitos/o-que-e-o-idh.html >. Acesso em: 27 de jan. 2020.

VEIGA, José Eli da. Desenvolvimento Sustentável - Desafio do Século XXI. Rio de Janeiro. Ed. Garamond, 2005. 200 p. 